\title{
Reflexiones sobre la pertinencia de la educación en el campo de la gerontología en Colombia y en el mundo*
}

\section{Reflections on education pertinence in the field of gerontology in Colombia and the world}

\author{
Lucelly Ardila Vega** \\ César Augusto Gómez Santos *** \\ Leonardo Alberto Vega Umbasía****
}

\begin{abstract}
**Magister en Comunicación Educativa, Gerontóloga, docente investigadora del programa de Gerontología de la Universidad del Quindío e integrante del Grupo de Investigación en Desarro1lo. Armenia, Colombia. lucellyardila@uniquindio.edu.co
\end{abstract}

***Magister en Economía Social y Dirección de Entidades sin Ánimo de Lucro, Gerontólogo, docente investigador del programa de Gerontología de la Universidad del Quindío e integrante del Grupo de Investigación en Desarrollo. Armenia, Colombia. cegomez@uniquindio.edu.co

****Magister en Territorio, Conflicto y Cultura, Antropólogo, Administrador Público, docente investigador del programa de Gerontología de la Universidad del Quindío y líder del Grupo de Investigación en Desarrollo. Armenia, Colombia. leonardovega@uniquindio.edu.co

Cómo citar: Ardila, L; Gómez, C.A \& Vega L.A. (2016) Reflexiones sobre la pertienencia de la educación en el campo de la gerontología en Colombia y en el mundo. Sophia 12(1): 71-84

\section{Resumen}

Este artículo tiene como propósito presentar un cúmulo de reflexiones sobre la pertinencia de la gerontología como carrera profesional, las cuales se sustentan en un conjunto de necesidades y aspectos que tienen resonancia a diferente escala (mundial y nacional) tales como: el envejecimiento demográfico y sus consecuencias; la transición epidemiológica; el perfil social de la población vieja; el reto de la dependencia; el rol de las personas mayores en la socialización de generaciones más jóvenes y, en la normatividad en temas relacionados con el envejecimiento y la vejez. Se concluye, con preocupación, que ante el panorama que justifica plenamente la necesidad de formar profesionales en gerontología, hoy exista una muy baja oferta de formación en pregrado en este campo en Colombia, lo cual demuestra la poca visibilización de las profundas transformaciones y consecuencias que, a mediano y largo plazo, trae el proceso de envejecimiento que actualmente vive el mundo y nuestro país.

Palabras Clave: Educación, envejecimiento, gerontología, vejez, universidad

\footnotetext{
*Artículo de reflexión derivado del trabajo realizado por parte de un equipo de docentes investigadores del Grupo de Investigación en Desarrollo, reconocido en categoría C por Colciencias, del Programa de Gerontología de la Universidad del Quindío, en torno a la necesidad de justificar la existencia y continuidad en la oferta de un programa de pregrado en el campo de la gerontología en Colombia. Se destaca y agradece la participación de otros docentes, en particular, Néstor Giraldo Navia y Leonardo Quintana Urrea.
} 


\begin{abstract}
This article presents a number of reflections on gerontology pertinence as a professional career, supported on a number of needs and aspects of world and domestic interest, such as: Demographic aging and its consequences; epidemiological transition; social profile of elder population; the challenge of independence; elders role in socialization of younger population, and regulations on matters related to aging and oldness. It is concluded with concern, that before the panorama fully justifying the need of educating professional of gerontology, there is a low offer of undergraduate formation in this field in Colombia, which demonstrates the scarce view of deep transformations and consequences brought, in the middle and in the long term, by the aging process currently faced by our country and the world.
\end{abstract}

Keywords: Education, aging, gerontology, oldness, university.

\section{Introducción}

Desde los años ochenta del siglo XX se tiene conocimiento de la existencia de programas académicos dedicados a la formación de gerontólogos en Colombia. La doctora Elisa Dulcey Ruíz (2001), afirmó que en esta década, el ámbito de la gerontología se caracterizó por un importante énfasis en la educación, la investigación y los servicios orientados a las personas mayores.

Esta misma autora indicó que en 1983 se abrió el primer programa de pregrado en gerontología en la Universidad Católica de Oriente (Rionegro), en el departamento de Antioquia, del cual con el tiempo se derivaron, a manera de extensión, otros programas de igual modalidad y con los mismos fines (mejorar las condiciones de vida para una vejez con calidad), en las universidades de San Buenaventura en Cali y Bogotá. Además reseñan que a mediados de esta misma década se abrieron otros dos programas: uno en Medellín, en el hoy conocido Instituto Tecnológico de Antioquia (gubernamental, antes Instituto Central Femenino), denominado Programa de Tecnología en Gerontología, con propósitos de formación y servicio; y otro en Armenia, en la Universidad del Quindío como programa de pregrado con un nivel de formación profesional. ${ }^{1}$ En la actualidad, estos programas de universidades públicas y privadas tienen presencia únicamente en los departamentos de Antioquia y Quindío.

\footnotetext{
1.Este programa inició labores académicas en el II semestre de 1987 y ha tenido hasta el momento cinco diseños curriculares. El primero va desde sus inicios hasta 1990; el segundo se implementa desde 1990 hasta 1997; el tercero desde 1997 hasta el 2004; el cuarto desde el 2004 hasta el 2007 año en el cual el Ministerio de Educación Nacional negó el registro calificado. El último diseño corresponde al nuevo programa el cual obtuvo su registro calificado por 7 años en diciembre de 2012 .
}

Para el año 2008, el SENA realizó un estudio para la caracterización de la gerontología en Colombia desde las competencias laborales y en diferentes dimensiones: organizacional, económica, tecnológica, ocupacional y educativa. Para efectos del presente artículo, se destaca lo concerniente a una de las tendencias futuras del entorno educativo, en el que se concluyó y se destacó la necesidad de la formación integral para el manejo de las consecuencias y efectos producidos por el envejecimiento individual y colectivo. (Sena, 2008: 140-141).

\section{Lagerontología, una disciplina emergente}

El interés por el estudio del envejecimiento y la vejez no es una cuestión reciente, por el contrario, este ha sido un tema que ha inquietado al hombre a lo largo de toda la historia de la humanidad; pero solamente hasta mediados del siglo XX, dado el evidente aumento de la población adulta mayor, en virtud de los avances de la medicina y la subsecuente prolongación de la esperanza de vida, se acentúan los esfuerzos por comprender académicamente los problemas de la vejez y el envejecimiento.

Lowenstein (2004), distingue tres fases en el desarrollo disciplinar de la gerontología, cada una de estas presenta ciertas particularidades que explican las características de la gerontología en su momento.

La primera fase, (fase temprana), está comprendida aproximadamente entre la década del 40 al 60. Se desarrolló cuando comienza a hacerse evidente la presencia de un fenómeno de extrema importancia: el envejecimiento de la población. Esto sucede aproximadamente a partir de la segunda mitad del siglo $\mathrm{XX}$. 
La gerontología nace para dar respuestas a las implicaciones demográficas, problemas de salud, económicos y sociales que ocasiona el fenómeno del envejecimiento. Por lo dicho, se puede afirmar que la gerontología en sus inicios presentaba una orientación funcionalista.

Esta etapa se caracterizó porque a nivel macro se interesó de manera especial por los aspectos demográficos del envejecimiento, su desarrollo teórico se fundamentó sobre la base de otras disciplinas, (perspectiva disciplinaria), con el propósito de adaptarse a condiciones sociales, en este punto empieza a presentarse un encuentro de campos que se esfuerzan por comprender mejor los problemas de la vejez; y en el nivel micro, esta fase estuvo marcada por una descripción de los aspectos culturales de los individuos viejos.

Debido a que en esta primera fase la gerontología surgió como un intento para dar respuesta a problemas de la vejez, la gerontología se interesó de manera importante por aspectos negativos del envejecimiento: soledad, aislamiento, pobreza, pérdidas, duelos, jubilación y una visión medicalizada del envejecimiento.

A partir de la Segunda Guerra Mundial comienza entonces a observarse una mejor definición de la gerontología, la cual se va configurando cada vez más como una disciplina interdisciplinar que tiene como objetivo el estudio del envejecimiento.

La segunda fase del desarrollo de la gerontología está comprendida aproximadamente entre las décadas del 60 al 80. Esta fase acepta la idea del envejecimiento como un proceso y se constituye como el objeto de conocimiento de la gerontología. Adopta la representación de que el envejecimiento es universal pero puede ser intervenido. En esta etapa se da una importante apertura de facultades de formación en gerontología (profesores, estudiantes, investigadores), en una variedad de programas de educación y entrenamiento en gerontología. En el nivel macro empiezan a surgir servicios y cuidados de larga duración, aplicados dentro de los servicios y modelos de atención de larga estancia, se presenta una demanda cada vez mayor de estos servicios (clientes). En el nivel mesocontextual la gerontología se desarrolla basada en matrices y modelos disciplinares, biológicas, psicológicas y sociales, es decir, se torna multidisciplinaria.

La tercera fase comprende el período de tiempo entre la década de los 90 hasta la fecha, en ésta se pasa de los modelos estructuralistas hacia patrones de inequidad social y metodologías interpretativas y constructivas, marcos de referencia interculturales, entre otros. Se ve la ciencia como una, (entre muchas), maneras de explicar los eventos. La gerontología empieza a ser concebida como un campo interdisciplinario. Se caracteriza por una importante proliferación de programas académicos de formación en gerontología en el mundo. En esta fase, la profesión alcanza un reconocimiento internacional.

Como hemos visto hasta aquí, la gerontología ha pasado por diversos momentos en su desarrollo disciplinar, en los que el objeto de estudio se ha ido transformando; primero el interés recayó en el desarrollo teórico y la práctica profesional centrado en las personas mayores, luego además de la personas mayores el interés se amplió al proceso del envejecimiento y más recientemente se ha desarrollado investigación en qué cambios se le deben atribuir al paso del tiempo.

Treas y Passuth (1988) propusieron un marco conceptual para el desarrollo futuro de la gerontología, lo denominaron modelo integrador. Los autores basan su propuesta en tres temas centrales que definen la misión y el propósito de la gerontología contemporánea: la edad, que representa una dimensión episódica del proceso de la vida, reconociendo que los acontecimientos suelen ocurrir o no en momentos específicos durante el ciclo de vida; el envejecimiento, que representa el paso dinámico del tiempo y las personas adultas mayores, el cual describe a las personas de edad que la sociedad define como viejas. Treas y Passuth Sugieren que estos temas están vinculados conceptualmente a la labor de las disciplinas de origen de la gerontología, (biología del envejecimiento, psicología del envejecimiento y sociología del envejecimiento), son útiles en la organización de nuestra comprensión de la gerontología como disciplina única. 


\section{Las necesidades de formación en el campo de la gerontología en el país y en el mundo}

Hoy existe en Colombia y en el mundo un panorama demográfico, epidemiológico, social, económico y normativo, que permite evidenciar un conjunto de necesidades y aspectos que justifican la formación de gerontólogos que puedan entender estas profundas transformaciones y sus consecuencias, que estén en capacidad de actuar profesional e integralmente en diversos contextos locales, nacionales $\mathrm{e}$ internacionales.

\section{EI envejecimiento demográfico}

Desde hace más de una década, la Organización Mundial de la Salud (OMS) ha señalado que en el año 2025 habrá más de 1.000 millones de personas con más de 60 años de edad en el mundo y se prevé que esta cifra se duplicará a casi 2.000 millones hacia el año 2050, con un efecto significativo: "En los países menos desarrollados donde el número de personas mayores pasará de 400 millones en 2000 a 1.700 millones en 2050" (OMS, 2004:1).

En este sentido, puede afirmarse que el mundo vive un proceso de envejecimiento global sin precedentes, de ahí que organismos como Unesco, ONU, OMS y OPS señalen al unísono, la importancia de asegurar un envejecimiento activo para todas las edades o como bien fue planteado por la OMS en el Día Mundial de la Salud, la buena salud a lo largo de la vida puede añadir vida a los años. Este fenómeno, que se percibe como una alteración de las estructuras demográficas de los países del mundo, se encuentra asociado a la disminución de las tasas de fecundidad y al aumento significativo de la esperanza de vida, lo que ha puesto en cuestión no solo las: "Ideas sobre la manera de financiar la asistencia a las personas mayores", sino también: "Las actitudes ante el envejecimiento" (OMS, 2012).

De acuerdo con la reciente publicación del Índice Global de Envejecimiento (2015), el cual mide el bienestar social y económico de las personas mayores, en el mundo en el año 2015, habían 102 países clasificados como sociedades jóvenes (menos de $10 \%$ de la población total tiene 60 y más años);
52 países son sociedades en transición (entre 10 y $19 \%$ de la población total tiene 60 y más años); 45 países son sociedades envejecidas (entre 20 y $29 \%$ de la población total tiene 60 y más años) y 1 país (Japón) es una sociedad hiperenvejecida (más del $30 \%$ de la población total tiene 60 y más años). Para el año 2030, se presentará una significativa reducción de los países que son sociedades jóvenes, pues serán 67, y habrá un aumento importante de las sociedades hiperenvejecidas, en 26 países. En el año 2050, solo 37 países serán sociedades jóvenes, 45 sociedades en transición, 56 sociedades envejecidas y 62 sociedades hiperenvejecidas. (HelpAge Internacional, 2015).

Para el caso de América Latina, la OMS hace 10 años, había señalado que esta región no era ajena a este fenómeno, pues la población que, en términos de longevidad, era de 547 millones en el 2005, llegará a 763 millones en el 2050. Esto se debe, entre otros factores, a que en los últimos 60 años se ha ganado en promedio 21.6 años de vida, pues la esperanza de vida que era de 51.8 años en 1955, pasó a 73.4 años en la actualidad. Como puntualizó Marcela Suazo en ese momento, directora de la división para América Latina y el Caribe del Fondo de Población de las Naciones Unidas: "Es una media ocho años más alta que la del total de las regiones en desarrollo y sólo 1.2 años menos que la de hoy en Europa" (El Tiempo, 2008: 1-10 Internacional).

En el año 2012, la OPS y la OMS señalaron que para el año 2020 las américas tendrán 200 millones de personas mayores, casi el doble que en 2006 y con más de la mitad viviendo en América Latina y el Caribe. Para el 2025, el 69\% de quienes nacen en Norteamérica y el 50\% de los que nacen en América Latina vivirán más allá de los 80 años (OPS/OMS, 2012):

El cambio demográfico será más rápido e intenso en los países con ingresos bajos y medianos. Por ejemplo, fueron necesarios 100 años para que el porcentaje de la población francesa mayor de 65 años pasara del 7\% al 14\%, [de similar manera], países como Brasil y China tardarán menos de 25 años en alcanzar el mismo crecimiento (OMS, 2012:1). 
El comportamiento descrito, se produce como resultado de la transición demográfica. Por mucho tiempo lo que aumentaba o mantenía un peso relativo alto era la población infantil, en tanto que las personas que llegaban a la edad adulta mayor era mínima. Lo anterior se corrobora con el estrechamiento progresivo de la pirámide poblacional, con ampliación simultánea de la punta, debido a la disminución de la población joven y al incremento de las personas mayores.

No en vano, la directora de OPS/OMS Mirta Roses Periago, ante los desafíos del envejecimiento de la población, se permitió afirmar hace algunos años: "El sector educativo debe desarrollar recursos humanos necesarios para afrontar esta realidad. Dijo: "En la región tenemos siete veces más pediatras que gerontólogos y todos debemos ajustarnos a la nueva realidad demográfica en todos los sectores" (Ministerio de Salud, 2012:1).

El Centro Latinoamericano y Caribeño de Demografía (Celade), en el año 2009, indicó que los países de la región se encuentran en distintas etapas del proceso de envejecimiento demográfico. Se observan cuatro fases: i) envejecimiento incipiente, ii) envejecimiento moderado, iii) envejecimiento moderadamente avanzado y iv) envejecimiento avanzado. Colombia se halla en el segundo grupo, el cual se caracteriza por tener tasas de fecundidad bajas (entre 3 y 2,3 hijos por mujer) y un índice de envejecimiento que oscila entre el 20\% y el 32\% (Cepal-Celade-FPNU, 2009:17, citado por Vega, 2014).

El porcentaje de población de 60 años y más en relación con el total de la población muestra que, para el caso de Colombia, en 1950 era del 5\%, en 1975 el $5.6 \%$, en el 2000 del $6.7 \%$, se estima que en el 2025 será el $14.6 \%$ y en el 2050 podría llegar al 23.9\%. El periodo 2000-2025 es el que presenta un mayor incremento, pues según las proyecciones del Celade, en este lapso Colombia, junto con Guayana Francesa, Costa Rica y Venezuela, se encuentra dentro del conjunto de países que presenta mayor incremento de la población de 60 años y más, con tasas superiores al 4\% (Cepal-Celade-FPNU, 2009, citado por Vega, 2014).

De acuerdo con la Encuesta Nacional de Demografía y Salud (ENDS) del año 2010, en el país, las personas mayores de 60 años representaban el $10 \%$ de la población y las personas de 65 años un 7\%: "Entre las ENDS de 1990 y de 2010, la proporción de población de menores de 15 años en Colombia bajó de 35 a 31 por ciento, en tanto que la población con 65 años y más aumentó de 5 a 7 por ciento" (Profamilia, 2011:17).

Diversos autores y organizaciones como Del Popolo (2001), Céspedes (2003), Fajardo y Rincón (2003), Jelin (2005), ONU (2006) y Profamilia (2011), han indicado que las principales causas del envejecimiento poblacional en la región y en Colombia son: el aumento de la esperanza de vida; el descenso de las tasas de fecundidad; los procesos de migración, en particular de la población adulta joven; el desplazamiento interno; y la disminución de la mortalidad, debido principalmente al control de las enfermedades infecciosas y parasitarias.

\section{La transición epidemiológica}

La inclusión en el análisis demográfico de la dinámica de la morbilidad, a través de la Transición Epidemiológica (TE), concepto creado en 1971 por Omran y ampliado por Lerner en 1973, derivado de la teoría de la transición demográfica, sugiere que esta sea tratada como un proceso continuo en el cual los patrones de salud y enfermedad de una sociedad se van transformando en respuesta a cambios más amplios de carácter demográfico, socioeconómico, tecnológico, político, cultural y biológico (Suárez, 1993 y Frenk, Bobadilla, Sepúlveda y López, 1996).

La reducción inicial en la mortalidad se concentra selectivamente en las causas de muerte de tipo infeccioso y tiende a afectar los grupos de edades más jóvenes, en los que las infecciones son más frecuentes y graves. Además, la supervivencia progresiva más allá de la infancia aumenta el grado de exposición a factores de riesgo asociados con enfermedades crónicas y lesiones, que incrementan así su contribución relativa a la mortalidad.

El posterior descenso en la fecundidad afecta la estructura por edades y repercute sobre el perfil de morbilidad, pues la proporción creciente de personas de edad avanzada aumenta la importancia de los padecimientos crónicos y degenerativos. Por tanto, se 
produce una nueva dirección de cambio, en la medida que la carga principal de muertes y enfermedades se desplaza de los grupos etarios más jóvenes a los de edades más avanzadas.

Después, emerge otro cambio en este proceso, dado por la disminución de la mortalidad y su sustitución por la morbilidad (aparición de enfermedades crónicas discapacitantes) como fuerza predominante que afecta notablemente el sistema de salud. El concepto de transición epidemiológica va más allá del de transición demográfica, este no solo intenta explicar los cambios en la mortalidad, sino también en la morbilidad. Así, en el proceso de transición, el significado de la enfermedad sufre una transformación radical: deja de ser primordialmente un proceso agudo que con frecuencia termina en la muerte, para convertirse en un estado crónico en el que muchas personas, en su mayoría de edad avanzada, sufren durante períodos largos de su vida. De este modo, se hace posible la combinación, aparentemente paradójica, de una mortalidad descendente junto con una morbilidad creciente (Brayne y Calloway, 1990).

Las modificaciones mencionadas en la estructura de la mortalidad por edad y por causas constituyen manifestaciones de la denominada "transición epidemiológica," que se debe a su vez, en gran medida a la transición demográfica y sus determinantes sociales. Se trata de la serie de cambios complejos e interrelacionados entre sí en los patrones de salud y enfermedad que ocurren en las poblaciones humanas a través de períodos más o menos prolongados. Normalmente suceden en una secuencia que va de un patrón dominado por las enfermedades infecciosas y de la primera infancia, asociados con pobreza, hacinamiento, desnutrición y falta de asistencia médica, a un patrón donde predominan las enfermedades crónicas.

En el caso de Colombia, como en la mayoría de los países de ingresos medios, el proceso de transición no ha seguido un curso lineal. Por el contrario, no hay una secuencia cronológica entre la disminución de las llamadas "enfermedades de la pobreza" y el aumento de las mal llamadas "enfermedades de la civilización". Ambas coexisten y dan lugar a la "transición prolongada y polarizada" (Frenk, Bobadilla, Sepúlveda y López 1996).
En relación con la sobrecarga del sistema de salud por enfermedades susceptibles de prevención, como una consecuencia de la transición epidemiológica, ya desde el año 1979, la OMS identificó las principales causas de mortalidad en el mundo para la cohorte de 65 años y más: las enfermedades del corazón, las cerebro-vasculares, los cánceres, la gripe y la neumonía, el enfisema y el asma, y la bronquitis (San Martín y Pastor, 1991). Para 1983 la OMS reportó como primera causa de morbilidad las enfermedades cardiovasculares (25\%); en segundo lugar, las enfermedades del aparato respiratorio (18\%); en tercer lugar, las enfermedades infecciosas y parasitarias (14\%) y en cuarto lugar los cánceres $(9 \%)$.

La Organización Panamericana de la Salud (OPS), citando a Jenkins (2005), afirmó que se ha demostrado la existencia de factores no genéticos que contribuyen a los 10 primeros diagnósticos de mortalidad y son factores de riesgo en todo el mundo, aunque su impacto varía en función de los ambientes y culturas locales. Esas causas de muerte son: consumo de tabaco; nutrición insuficiente o excesiva (hábitos alimentarios); ejercicio aeróbico insuficiente; consumo excesivo de alcohol; falta de inmunización contra agentes microbianos; exposición a toxinas y venenos; armas de fuego; comportamientos sexuales arriesgados; traumatismos por vehículos automotores; y consumo de drogas ilegales.

En Colombia, según el DANE (2008) las diez primeras causas de defunción en su orden son: infarto agudo de miocardio; agresión con disparo de otras armas fuego y las no especificadas; otras enfermedades pulmonares obstructivas crónicas; neumonía organismo no especificado; diabetes mellitus no especificada; tumor maligno del estómago; otras enfermedades cerebro vasculares; tumor maligno de los bronquios y del pulmón; enfermedad cardiaca hipertensiva; $y$ hemorragia intraencefálica.

Es fácil concluir que las personas mayores se convierten en los usuarios que más acuden al sistema de salud a consecuencia del incremento en las enfermedades prevalentes y dependientes con la edad (Gómez y Curcio, 2002), a pesar que estas, son susceptibles de prevención o minimización de sus secuelas en un alto porcentaje, o en los casos menos favorables, al menos es posible postergar sus manifestaciones más severas. 
En la actualidad, desde la perspectiva de Lalonde (1990), el campo de la salud se divide en cuatro componentes que es necesario tener en cuenta en cualquier intento por mejorarla. Estos son: biología humana, medio ambiente, estilo de vida y organización de la atención en salud y tienen relación directa con los determinantes de la salud planteados por Blumm (1960).

El componente de biología humana incluye todos los hechos relacionados con la salud física y mental, que se manifiestan en el organismo como consecuencia de la biología fundamental del ser humano y de la constitución orgánica del individuo. De él hace parte la herencia genética de la persona, los procesos de maduración y de envejecimiento y los diferentes aparatos internos del organismo. Dada la complejidad del cuerpo humano, su biología puede repercutir sobre la salud de maneras múltiples, variadas y graves, además de que puede fallar de diversos modos. Este elemento contribuye a la mortalidad y a toda una gama de problemas de salud, cuyas causas radican en la biología humana y generan altísimos costos, de miles de millones de pesos, en los tratamientos.

El medio ambiente que incluye todos aquellos factores relacionados con la salud que son externos al cuerpo humano y sobre los cuales la persona tiene poco o ningún control.

El estilo de vida como componente del campo de la salud, representa el conjunto de decisiones que toma el individuo y sobre las cuales ejerce cierto grado de control. Las malas decisiones y los hábitos personales perjudiciales conllevan riesgos que se originan en el propio individuo. Cuando esos peligros tienen como consecuencia la enfermedad o la muerte, se puede afirmar que el estilo de vida de la persona contribuyó a ellas.

La cuarta categoría del concepto, la organización de la atención de la salud consiste en la cantidad, calidad, orden, índole y relaciones entre las personas y los recursos en la prestación de servicios de atención, también se define como sistema de atención de la salud. Es aquí en donde generalmente se orientan los esfuerzos de la sociedad para mejorarla y es este componente el que genera la mayor parte de los gastos directos en esta materia, sin embargo, cuando se identifican las causas principales de la enfermedad y de la muerte, se concluye que su origen está en los otros tres componentes del concepto. Es decir, se están gastando grandes cantidades de dinero en tratar enfermedades que, desde un principio, podrían haberse prevenido.

Es por esta razón que para aportar desde la gerontología al campo de la salud y del envejecimiento activo, se debe orientar la formación hacia la comprensión y promoción de modelos de intervención que estén más próximos a los tres primeros elementos del concepto del campo de la salud, con el propósito de prevenir la enfermedad, promover la salud y reducir la discapacidad.

El problema de la no promoción de la salud desde etapas tempranas se refleja a largo plazo y específicamente en las generaciones maduras, generando dependencia, y esta se convierte en un riesgo social más que de salud, ya que el estado físico real de las personas muchas veces refleja fuertes componentes sociales. En Colombia los mayores han acumulado una serie de desventajas en las que su vulnerabilidad física y social aumenta drásticamente, debido a que los escasos recursos educativos y económicos reducen la capacidad de adaptación y de afrontar sus propias limitaciones, lo que se traduce en demandas de protección y ayuda.

La Organización Mundial de la Salud definió el envejecimiento activo: "Como el proceso de optimización de las oportunidades de salud, participación y seguridad con el fin de mejorar la calidad de vida a medida que las personas envejecen" (OMS, 2002:1). El planteamiento de envejecimiento activo se basa en el reconocimiento de los derechos humanos de las personas mayores a la igualdad de oportunidades y en los Principios de las Naciones Unidas a favor de las personas de edad.

Para la OMS los orígenes del riesgo de las enfermedades crónicas comienzan en la primera infancia, más tarde el riesgo es determinado y modificado por factores, como la situación económica y las experiencias durante toda la vida y el riesgo aumenta en la medida que las personas envejecen, de ahí, la importancia de afrontar los riesgos desde edades tempranas y a lo largo de todo el ciclo vital, ya 
que las enfermedades no trasmisibles se convierten en las principales causas de morbilidad, discapacidad y mortalidad en todas las regiones del mundo.

El enfoque del envejecimiento activo en las políticas y programas de desarrollo ofrece la posibilidad de afrontar muchos de los retos tanto de las personas como de las poblaciones que están envejeciendo. Por ello, el marco político para el envejecimiento requiere la acción sobre tres pilares básicos: la salud, la participación y la seguridad.

En cuanto a salud se debe: primero, prevenir y reducir la carga del exceso de enfermedades crónicas, discapacidades y mortalidad prematura; segundo, reducir los factores de riesgo de enfermedades importantes y aumentar los componentes que protegen la salud durante todo el ciclo vital; tercero, desarrollar una continuidad de servicios sociales y de salud accesibles de calidad y respeto por la edad; y cuarto, proporcionar formación y educación a los cuidadores.

En lo referente a la participación se trata de: primero, proporcionar oportunidades de educación y aprendizaje durante toda la vida; segundo, reconocer y permitir la participación de las personas en las actividades de desarrollo económico, en la fuerza laboral, en espacios de voluntariado de acuerdo con sus necesidades y preferencias; $y$ tercero, alentar a las personas a participar plenamente de la vida familiar a medida que envejecen.

En cuanto a seguridad, se contempla: primero, asegurar la protección y la dignidad de las personas mayores, abordando sus derechos y las necesidades de seguridad social, financiera y física; segundo, reducir las desigualdades en los derechos de seguridad y las necesidades de las mujeres mayores.

\section{Perfil social de la población vieja en Colombia}

Cowgill (1974, citado por Mishara 1986), relacionó la condición social de las personas de edad con los cambios sociales y en este sentido identificó cuatro tendencias que han contribuido al descenso de la condición de las personas mayores en el desarrollo de la sociedad moderna. Estas son: perfeccionamiento en la tecnología sanitaria, desarrollo de la tecnología moderna, urbanización e instrucción.

Aunque los planteamientos de este modelo los expuso Cowgill con base en los países desarrollados, los naciones en desarrollo, en este caso Colombia, también han sufrido un proceso de transformación y han logrado un crecimiento económico global que les ha permitido ampliar la cobertura en sectores como la salud, la educación y la industria, pero al mismo tiempo ha traído como consecuencia una competencia entre generaciones, que se traduce en menos oportunidades para la población vieja, pues los nuevos roles otorgan superioridad en las esferas productivas y de conocimiento a las generaciones más jóvenes.

En la relación crecimiento $\rightarrow$ producción $\rightarrow$ consumo $\rightarrow$ satisfacción, el cambio lo comporta el desarrollo de las fuerzas productivas, trayendo como consecuencia la sobreestimación de la capacidad productiva de los jóvenes trabajadores, y la subvaloración de las personas mayores, produciéndose así, una mayor discriminación por la edad.

La vejez como categoría social en Colombia refleja una realidad en la que las personas mayores se enfrentan a una situación de pobreza, miseria y poca participación social y laboral, primando un concepto asistencialista en los programas y servicios dirigidos a este colectivo. El hecho de que un alto porcentaje de la población de 50 años y más, no tenga pensión ni recursos para su sustento, agrava más su realidad y acentúa su situación de riesgo y vulnerabilidad.

Salazar (2004) con base en la Encuesta Nacional de Hogares (ENH) de 1996 a 1999, infiere que la población vieja en Colombia presenta un bajo perfil social. Como fuerza de trabajo, es un grupo etario marginado; en un alto porcentaje es inactivo y las mujeres presentan porcentajes más altos que los hombres. La población activa en lo económico deriva su sustento de la acción en el sector informal y sus ingresos oscilan entre uno y dos salarios mínimos. Presenta bajos niveles de escolaridad y la mayoría está excluida del sistema de seguridad social en pensiones. 
En este panorama, aunque la mayoría de la población de personas mayores está en condiciones desventajosas, los que están en la indigencia o muy cercana a ella, no lo son por su condición de viejos, sino por su preexistente posición de ciudadanos marginados.

Al lado del empobrecimiento económico hay también un fenómeno de "visión pauperizada y pauperizadora" del proceso de envejecimiento y la vejez, lo que se manifiesta en la presencia de representaciones sociales, estereotipos y prejuicios que evidencian en la población la configuración, transmisión y replicación de un "modelo deficitario", que se manifiesta en: una creciente disminución del rendimiento intelectual a lo largo del ciclo vital; una pérdida del rendimiento laboral; una merma de la capacidad para el erotismo, la intimidad, el amor, el disfrute de la sexualidad y la genitalidad y un empobrecimiento de la personalidad, por un detrimento del interés en lo que las rodea, insuficiente interacción social, poca participación, "depresión”, terquedad, intransigencia.

\section{El reto de la dependencia}

La dependencia funcional de las personas mayores es un problema creciente en los países desarrollados y dentro de poco se sentirá con fuerza en los que están en desarrollo. Para lograr reducir la carga de enfermedad y crear las condiciones que la modifiquen en un futuro, se deben desarrollar acciones inmediatas, ya que su envejecimiento poblacional se está dando en un contexto social marcado por las desigualdades sociales.

Con base en lo expuesto en el punto anterior, y apoyados en Maravall (1997), puede concluirse:

Que históricamente la vejez ha sido para la mayor parte de la población sinónimo de pobreza en lo económico, inactividad en lo laboral, marginalidad en la vida social y cultural, enfermedad en el ámbito de la salud. Las personas quedaban, por tanto, a expensas de los cuidados que sus familiares quisieran $\mathrm{y}$ pudieran darles (p. 1).

La presentación o agudización de situaciones de deterioro físico, sensorial o psíquico afectan la vida independiente, provocan cambios en las relaciones familiares e intergeneracionales, reducen las posibilidades de vida autónoma y exigen unas medidas de atención externa, e indispensable que constituyen una respuesta al reto de la dependencia. Si en países desarrollados, que cuentan con mayor trayectoria en reflexión y respuestas a estos problemas, se considera que la reacción pública a esta problemática es "tardía e insuficiente" ¿Qué podría decirse de países como Colombia?

La problemática esbozada, estudiada y descrita por investigadores y trabajadores de la salud, la educación, la planeación y campos afines ha originado una creciente conciencia, compartida en mayor o menor grado por todos, en el sentido que existe una necesidad perentoria de iniciar tempranamente en el ciclo vital de cada cohorte de la población, acciones eficaces para facilitar en cada persona el desarrollo de:

\section{Competencias para la vida}

- Conciencia de la responsabilidad de la propia salud y adopción de estilos de vida saludables, hábitos sanos y estrategias de autocuidado y prevención desde etapas tempranas del ciclo vital.

- Un proyecto de vida personal coherente con el de la propia familia.

- Una autogestión del propio envejecimiento no solo en cuanto a la salud sino también en lo laboral, lo económico y lo social con un plan de retiro a largo, mediano y corto plazo que posibilite autosuficiencia para una vejez digna.

- Una visión del proceso de envejecimiento con una manifestación de la vida y su dinámica, por lo tanto, como un proceso normal que no está ligado a la enfermedad y que aunque implique cambios e incluso limitaciones no es necesariamente deficitario y mucho menos empobrecedor del potencial básico. Por el contrario, puede y debe, ser asumido como una oportunidad de crecimiento y desarrollo personal.

Desde el punto de vista biológico es necesario advertir que la constante prolongación de la esperanza de vida, determina que conforme aumenta el tiempo total de vida, las personas mayores viven más años 
con mala salud, es decir, los individuos que sufren de enfermedades crónicas progresivas deben afrontar el reto de permanecer activas e independientes a pesar de sus problemas de salud. Dicho de otra manera, estamos viviendo un aumento significativo del número de años que es posible vivir desde el nacimiento hasta la muerte, pero sin un aumento correspondiente en la salud, en consecuencia se pasa una porción mucho más prolongada del curso de la vida con mala salud y en estado de dependencia.

\section{El rol de las personas mayores en la socialización de las generaciones más jóvenes}

De acuerdo con los nuevos escenarios presentes en la esfera familiar originados por los múltiples cambios sociales, económicos y culturales, que modificaron la estructura y funciones de esta institución, induciendo a que los abuelos compartan con sus nietos mayor tiempo, convirtiéndose en agentes de socialización significativos para los nietos y contribuyendo al desarrollo social, cognitivo, moral y afectivo de los menores, es importante recordar que las técnicas de socialización utilizadas por los agentes socializadores, influyen en la construcción de la identidad del sujeto en proceso de socialización y facilitan, o no, el desarrollo de su autonomía y la conciencia participativa y democrática, revertidas en las estructuras micro y macro sociales que rodean al individuo. La utilización de un estilo basado en el autoritarismo generará personas con bajos niveles de desarrollo moral, mientras que el uso de un estilo basado en la democracia, propiciará adultos con mayores niveles de desarrollo moral y con mayor independencia.

Estos aspectos han generado una conciencia mundial de la problemática asociada al envejecimiento $\mathrm{y}$ la vejez, materializada en un conjunto de eventos significativos realizados en el contexto internacional y regional, como: Encuentro de Haifa (Israel, 1982), asambleas mundiales sobre el envejecimiento, (Viena 1982 y Madrid 2002), conferencias regionales (I Conferencia Latinoamericana y del Caribe, Bogotá 1986), además de congresos, simposios y seminarios en diferentes latitudes. Nuestro país no ha estado ajeno a este fenómeno y el mismo gobierno nacional ha tratado de esbozar una política que se expresa en una normatividad, pero, infortunadamente, en muchas de ellas no van más allá de la expresión de buenas intenciones.

\section{Normatividad en materia de envejecimiento y vejez en Colombia}

Desde el siglo XIX, el Estado y, en particular, las diferentes comunidades religiosas y las juntas de beneficencia asumieron la responsabilidad de la asistencia social a las personas mayores. Sin embargo, para contextualizar este punto sobre la normatividad, tomamos como referente la promulgación de la nueva Constitución Política en el año de 1991, en donde nuestro país se asume como un estado social de derecho, lo cual ha llevado a que en las dos últimas décadas, se presente un desarrollo normativo considerable e importante en temas relacionados con la atención y protección a las personas mayores y asumir las implicaciones del envejecimiento poblacional.

De forma consecutiva a la legislación de protección y atención a las personas mayores, se ha venido promoviendo, desde el ámbito internacional, la necesidad de formar profesionales en gerontología que contribuyan a la prevención y atención de la problemática descrita.

En este sentido, desde el punto de vista normativo a partir del año 1993 se empezaron a dictar disposiciones en relación con el ejercicio del gerontólogo como profesional del campo de la salud. Al respecto se encontró cómo, mediante la Resolución 00449 del 8 de febrero de 1993, se delegó en los jefes de los servicios seccionales de salud, la función de inscribir o registrar en el área de su jurisdicción el título Profesional en Gerontología. Una vez efectuado el registro se relacionan ante el Ministerio de Salud.

En este escenario normativo, se sancionó la Ley 1164 del 3 de octubre de 2007, por la cual se dictan disposiciones en materia del talento humano en salud, y que reglamenta en su capítulo IV (Del ejercicio de las profesiones y de las ocupaciones del Talento Humano en Salud), artículo 17: 
Las profesiones del área de la salud están dirigidas a prestar atención integral en salud, la cual requiere la aplicación de las competencias adquiridas en los programas de educación superior en salud. A partir de la vigencia de la presente ley se consideran como profesionales del área de la salud, además de las ya clasificadas, aquellas que cumplan y demuestren a través de su estructura curricular y laboral, competencias para brindar atención en salud en los procesos de promoción, prevención, tratamiento, rehabilitación y paliación.

Producto de esta norma, se constituyó una mesa técnica de carácter nacional compuesta por gerontólogos de las diferentes facultades y programas del país, que ha venido construyendo desde el mes de febrero de 2008, las normas de competencia de la gerontología a nivel de pregrado, proceso que fue asesorado metodológicamente por el Sistema Nacional de Formación para el Trabajo (SNFT) del SENA y avalado por la Mesa Sectorial Nacional de Salud. Las normas de competencia que resultaron de este ejercicio, fueron sometidas a verificación y a revisión, mediante consulta pública realizada por los asesores metodológicos.

En el año 2013 se promulgó la Ley 1655 del 15 de julio que modificó el artículo 70 de la Ley 1276 de 2009 quedando así:

Gerontólogo. Profesional de la salud, titulado de instituciones de Educación Superior debidamente acreditadas para esta área específica del conocimiento, que interviene en el proceso de envejecimiento y vejez del ser humano como individuo y como colectividad, desde una perspectiva integral, con el objetivo de humanizar y dignificar la calidad de vida de la población adulta mayor (p. 1).

\section{Otras razones que justifican la oferta de programas de gerontología}

Además de las razones expuestas, se enumeran otras situaciones que justifican la necesidad de formar profesionales en gerontología en nuestro país. Entre otras se destacan las siguientes: la creación del Nodo Nacional de Investigación en Envejecimiento; la inclusión de líneas de investigación en envejecimiento y vejez en la Agenda Nacional de CTI de Colciencias (2010); la formulación de la Política Nacional de Envejecimiento y Vejez 2007-2019; los consensos logrados en los tres cursos de formación de docentes de gerontología en América Latina organizados por la Asociación Colombiana de Gerontología y Geriatría y realizados en las ciudades de Manizales (2009 y 2013) y Armenia (2011). El primer curso permitió hacer distinciones epistemológicas entre teoría, modelo y paradigma, se abordó el desarrollo de los principales modelos sociales de envejecimiento, con enfoque de curso de vida, esto permitió que los participantes se capacitaran en las bases conceptuales y en la descripción de los modelos sociales y psicológicos del envejecimiento con énfasis en las consecuencias psicosociales de este proceso a nivel individual y poblacional, para su difusión a través de la actividad docente. En el segundo curso se trabajaron ampliamente las temáticas de interdisciplinariedad, multidisciplinariedad y transdisciplinariedad. En el tercer curso, se compartió acerca de algunas bases conceptuales que permitan analizar la perspectiva de la vida, desde diferentes disciplinas, intercambiando puntos de vista teóricos e investigativos para hacer de la actividad docente gerontológica un proceso abierto a la actualización y al análisis permanente.

Por último, en las conclusiones de los congresos internacionales de universidades con carreras de gerontología realizados en Argentina (2010), México (2012) y Perú (2014) se hace un llamado para articular $\mathrm{y}$ fortalecer la formación de gerontólogos.

En Argentina, específicamente en la Universidad Maimónides de Buenos Aires, se marcó la pauta para empezar a reflexionar en conjunto sobre la formación de pregrado, una de las conclusiones fue la imperiosa necesidad de formar profesionales que puedan dar respuesta a las necesidades de los mayores con menos costo social y económico, con una concepción interdisciplinaria que facilite un enfoque integral de la problemática gerontológica, que no quede encerrada únicamente en lo médico, o en lo psicológico, o en lo social. Allí se tuvo la oportunidad de mostrar que no era solo Colombia la que formaba gerontólogos, que países como Panamá, Brasil, Venezuela, México y Perú también habían afrontado el reto de hacerle frente al envejecimiento demográfico. 
En México, la reflexión se centró básicamente en las buenas prácticas con el objetivo primordial de analizar y debatir sobre el estado de la praxis gerontológica, desde los actores en la formación profesional en América Latina, para generar líneas de acción.

En Perú se hizo un reconocimiento a los esfuerzos de las diferentes universidades para ofrecer una formación de calidad. Se conformó la Red Panamericana de Programas y/o carreras universitarias de gerontología con el objeto, en primera instancia, de formalizar convenios de índole académico, para el intercambio estudiantil y docente. Se destacó el papel de la investigación como parte fundamental en la construcción de un marco teórico, metodológico y epistemológico de la gerontología.

Continuando con la realización de estos congresos, el próximo se llevará en la Universidad Católica de Oriente en Rionegro (Antioquia) en octubre del 2016, llegando así a la cuarta versión, bajo el lema "Envejecimiento, Desarrollo Humano y Social, un Reto para el Gerontólogo". Hacia el Posicionamiento del Gerontólogo en el Ámbito Internacional.

\section{Conclusiones}

Ante este panorama que justifica plenamente la necesidad de formar profesionales en gerontología, es preocupante que hoy existan en Colombia solo dos programas a nivel profesional, uno ofrecido por la Universidad del Quindío modalidad presencial y otro por la Universidad Católica de Oriente de Rionegro (Antioquia) en modalidad distancia y con apoyo virtual. Existe además un programa de tecnología en gerontología ofrecido por el Instituto Tecnológico de Antioquia. A nivel de posgrados se destaca la trayectoria de la Maestría en Gerontología, Envejecimiento y Vejez de la Universidad de Caldas y una reciente especialización en Gerontología Social de la Universidad La Salle en Bogotá. Esta baja oferta de formación en el campo de la gerontología en nuestro país, demuestra la poca visibilización de las profundas transformaciones y consecuencias que a mediano y largo plazo trae el proceso de envejecimiento que actualmente vive el mundo.

Aunque en el mundo existe una diversidad de programas de postgrado en gerontología, en los últimos años ha ido aumentando la oferta de programas en el nivel de pregrado en algunos países de América Latina, en Estados Unidos, Canadá y Portugal; se ha encontrado que la duración de estos programas de formación están por debajo de la duración de los programas ofertados en Colombia.

Se destaca el papel, tal como lo advirtieron Lowenstein y Carment (2009 citados por Vega 2014), que deben empezar a tomar las instituciones educativas para facilitar la adaptación social frente al reto de las necesidades sociales y de salud relacionadas con el envejecimiento global. Así mismo, el desarrollo de los conocimientos y programas educativos en gerontología es uno de los medios esenciales para sensibilizar al público y los profesionales que trabajan con personas mayores frente al impacto de las sociedades que envejecen.

\section{Referencias bibliográficas}

Brayne C, Calloway P. (1990). The association of education and socioeconomic status with minimental state examination and the clinical diagnosis of dementia in elderly people. Age and Ageing; 19:91-6.

Cepal/Celade/FNUAP (2009). El envejecimiento y las personas de edad. Indicadores sociodemográficos para América Latina y el Caribe. Santiago de Chile: Centro Latinoamericano de Demografía-Celade y Fondo de Población de las Naciones Unidas -FNUAP.

Céspedes, E. (2003). El proceso de transición en salud en Colombia e implicaciones para el milenio. En La Cátedra abierta en población 2000-2001. Bogotá: Universidad Externado de Colombia y Fondo de Población de las Naciones Unidas. 2003.

Del Popolo F. (2001). Características sociodemográficas y socioeconómicas de las personas en América Latina. Cepal.

Dulcey, E. (2001) La sociedad civil, el envejecimiento y la vejez en Colombia. A propósito de relaciones de ONG. Bogotá. Recuperado de: http://www.facso.uchile.cl/observa/ sociedad_enveje.pdf. 
El Tiempo. (2008). La población latinoamericana envejece a una gran velocidad. 13 de junio de 2008, Bogotá. Recuperado de:http:// www.eltiempo.com/archivo/documento/ MAM-2972627.

Fajardo, M. y Rincón, M. J. (2003). Demografía del envejecimiento y sus implicaciones en sectores claves de la sociedad colombiana (pp. 57-102). En L. Wartenberg (Comp.). La cátedra abierta en población 2000-2001. Bogotá: Universidad Externado de Colombia y Fondo de Población de las Naciones Unidas.

Frenk, J; Bobadilla, J; Sepúlveda, J y López, M. (1996). Health transition in middle income countries: new challenges for health care. Health Pol Plan; 4: 26.

Gómez, J y Curcio, C. (2002). Valoración Integral de la Salud del Anciano. Editorial Tizan. Manizales.

HelpAge International. (2015). Índice Global de Envejecimiento. Resumen Ejecutivo. Recuperado de: http://www.helpage.es/ noticias/lanzamiento-del-ndice-global-sobre-envejecimiento-2015/.

Jelin, E. (2005) "Las familias latinoamericanas en el marco de las transformaciones globales. Hacia una nueva agenda de políticas públicas", En: Arriagada, I. Políticas hacia las familias, protección e inclusión sociales. Serie Seminarios y Conferencias, núm. 46. Comisión Económica para América Latina y El Caribe, Naciones Unidas, Santiago de Chile, Chile.

Jenkins, C.D. (2005). Mejoremos la salud a todas las edades. Un manual para el cambio de comportamiento. OPS.

Lalonde, M. A. (1990). A new perspective on the health of Canadians: a working document. Ottawa: Minister of Supply and Services Canada.
Lowenstein, A. (2004). Gerontology coming of age: the transformation of social gerontology into a distinct academic discipline. Educational Gerontology, 30, p. 129-141.

Maravall, H. (1997). "La atención a la dependencia: El gran reto de la política social hacia las personas mayores de los próximos años". Recuperado de: http://www.copmadrid.org/ webcopm/publicaciones/social/1997/vol1/ $\underline{\operatorname{artil.htm}}$

Ministerio de Salud. (2012). La salud es un tema que no debe ser de un día sino de toda la vida. Recuperado de:https://www.minsalud. gov.co/Paginas/La $\% 20$ salud $\% 20$ es $\% 20$ un $\% 20$ tema $\% 20$ que $\% 20$ no $\% 20$ debe $\% 20$ ser $\% 20 \mathrm{de} \% 20 \mathrm{un} \% 20 \mathrm{~d} \% \mathrm{C} 3 \% \mathrm{ADa} \% 20$ sino $\% 20$ de $\% 20$ toda $\% 201 \mathrm{a} \% 20$ vida $\% 20$ directora $\% 20 \mathrm{de} \% 20 \mathrm{OPS} \% 20 \mathrm{OMS} . \mathrm{aspx}$

Mishara, B.L; Riedel, R.G. (1986). El proceso de envejecimiento. España: Ediciones Morata.

Organización Mundial de la Salud OMS. (2002). Active Aging. Geneva: WHO.

Organización Mundial de la Salud OMS. (2004). Envejecimiento y ciclo de vida. Traslación del conocimiento. Recuperado de:http:// www.who.int/ageing/projects/knowledge translation/es/

Organización Mundial de la Salud (OMS). (2012). Día mundial de la salud 2012. ¿Está usted preparado? Recuperado de:http://www. who.int/world-health-day/2012/toolkit/ background/es/

Organización Panamericana de la Salud (OPS) Organización Mundial de la Salud (OMS). (2012). Vivirán más de 80 años más de la mitad de los que nazcan en las américas en la próxima década. Recuperado de: http:// www.paho.org/arg/index.php?option=com content \&view $=$ article \&id=929:viviranmas-80-anos-mas-mitad-que-nazcan-americas-proxima-decada\&Itemid $=290$ 
Profamilia (2011). Encuesta Nacional de Demografía y Salud ENDS 2010. Bogotá. Disponible en: https://dhsprogram.com/pubs/pdf/FR246/ FR246.pdf

Roses Periago, M. (2012). La salud es un tema que no debe ser de un día sino de toda la vida. Recuperado de: https://www.minsalud. gov.co/Paginas/La\%20salud $\% 20$ es $\% 20$ un $\% 20$ tema $\% 20$ que $\% 20$ no $\% 20$ debe $\% 20$ ser $\% 20 \mathrm{de} \% 20 \mathrm{un} \% 20 \mathrm{~d} \% \mathrm{C} 3 \% \mathrm{ADa} \% 20$ sino $\% 20 \mathrm{de} \% 20$ toda $\% 201 \mathrm{a} \% 20 \mathrm{vida} \% 20$ directora $\% 20 \mathrm{de} \% 200 \mathrm{OS} \% 20 \mathrm{OMS}$.aspx

Salazar, L. (2004). El Envejecimiento en Colombia - Una construcción social. Armenia: Universidad del Quindío: Mimeo.
Servicio Nacional de Aprendizaje -SENA-. (2008).Caracterización de la gerontología en Colombia. Una mirada desde las competencias. Bogotá: Sena.

Suárez R. (1993). Geriatría. ¿Nueva especialidad? Revista. Cubana Med. Gen. Intrg; 9: 6-8.

Treas, J y Passuth, P. (1988). "Age, Aging and the aged: The three siciologies". En E. F. Borgatta y K. Cook (Ed.) The future of sociology: Newbury Park. CA.

Vega, L.A. (2014). Ciudad, envejecimiento-vejez y educación: Elementos para develar un conflicto entre la ciudad concebida y la ciudad practicada. Universidad La Gran Colombia, Sophia. 10 (1), 50-63. 